\title{
The Role of Sleep and Impact on Brain and Learning
}

\author{
https://doi.org/10.3991/ijes.v8i3.17099 \\ Sevasti Kapsi $\left.{ }^{(}\right)$, Spyridoula Katsantoni, Athanasios Drigas \\ N.C.S.R. 'Demokritos', Athens, Greece \\ sevastikapsi@gmail.com
}

\begin{abstract}
There are many interventions which may enhance learning. Many techniques are used in education to empower memory, which is a basic cognitive ability to ensure learning. A question arises: if learning is a natural process, is there a natural mechanism which supports learning? In this review, it is supported that sleep is such a mechanism. Research results on sleep and learning are presented and support different effects on the brain and learning, according to the age of the population. Sleep is a fundamental process for brain function and cognition. More studies should follow to make good use of this information, so as to design new interventions for the field of education.
\end{abstract}

Keywords-Sleep, memory, learning, cognitive enhancement.

\section{Introduction}

According to the Greek mythology, Hypnos and Thanatos were twin brothers, the Night's and Erevos' children. They were assumed to be powerful gods and especially Hypnos was supposed to be the lord of all gods, as referred in Iliad, by Homer [1]. Hypnos had been worshipped in ancient Greece and had been considered to be really significant.

Sleep is a state of consciousness in which most senses are disengaged and a lack of responsiveness to the environment follows. The body is mostly motionless, visual contact is lost, while the reaction to sound becomes scattered. Short term memory stops transmitting information to long term memory and this pathway closes down. As one is falling asleep, memories of recent experience can be maintained until 3 minutes and then they fade away [2].

How is it possible for such a kind of physical state of mind and body to enhance cognition? Can sleep enhance learning? Has sleep been as important as it was supposed to be since the ancient years? Research supports that sleep and memory processing are tightly connected and they function in an interactive way. In fact, learning keeps going on, during sleep, in an offline mode [3].

Sleep contributes to basic physiological functions of the body regarding hormones, and body-temperature regulation, immunity system, ontogenesis, and tissue restoration. Sleep deprivation causes increase in blood pressure, tolerance in glucose, sensitivity in insulin, and diabetes. It can also produce developmental, cognitive and emotional disorders [4], [3]. 
The purpose of this study is to present research results on the way sleep contributes to brain function and learning. In particular, the review includes information about the learning process during sleep at different stages of sleep and different stages of development. Researchers mostly adopt sleep deprivation or sleep extension in the research protocols, to find out about the impact of sleep on learning. There are also many studies which support that memory consolidation is related to sleep. Memory is significant to learning especially in language learning and comprehension [5]. Finally, recommendations are made to empower learning through sleep, as well as suggestions about new research.

\section{Method}

The present study is a review of research articles on Google Scholar and on Mendeley, from 2000 to 2020. The research was made in English using keywords such as Hypnopaedia, sleep and learning, sleep and memory, sleep and cognition. Peer group studies that had been published in scientific journals, were mostly preferred.

Hypnopaedia refers to the enhancement of cognitive abilities through sleep mechanisms. The research results are presented in two sections: Firstly, results on the brain function during sleep are referred and secondly results on the impact of sleep on learning at different stages and ages come after. Lastly, conclusions are drawn.

\section{$3 \quad$ Sleep and Brain Function}

Sleep is a physiological state which varies in the different cycles of brain function. In fact, brain follows several periods of metabolic and neuronal changes which distinguish sleep from arousal [6].

In particular, there are two states which are dominant during sleep: NREM state (Non- Rapid Eye Movement) and REM state (Rapid Eye Movement). The difference between them is in the eye movement that can be observed externally. In addition to that, in NREM state electroencephalogram (EEG) shows orchestrated movements, sleep spindles, at $12-16 \mathrm{~Hz}, \mathrm{~K}$-complexes, slow brain wave movement, low muscle tone and minimum psychological activity. In this state there are four distinct stages which are repeated every 90 to 100 minutes. This distinction is based on EEG recorded differences in brain frequencies and brain wave speed. Sleep starts with NREM state with brainwaves of alpha frequency in the first stage and then lower frequencies of theta band follow in the second stage. The third stage of NREM state is very short and then the fourth stage follows rapidly in the delta band brainwave activity. Arousal is possible in the first stage, while in the other stages it is not [7], [6], [2], [8].

The REM state of sleep follows afterwards. It is defined by a disorganized EEG, toneless muscles, episodic eye movement, cardiovascular abnormality and dreaming state. The human body appears motionless along with an active brain. REM state lasts for 1 to 5 minutes. In the first half of night sleep Slow Wave Sleep frequencies are dominant in $80 \%$ of sleep. During this phase acetylcholine, noradrenaline, and cortisol 
are reduced. In the second half of the state of sleep, REM state increases (20-25\% of total sleep) and appears earlier in stage 2 of NREM state [7], [6], [2], [8]

Sleep is a combination of physiological and behavioral processes of inactivity and movement, concurrently, which are either visible or not discrete [2]. It is related to synaptic homeostasis and according to Tononi \& Cirelli [9], during arousal, synapses are charged, while during sleep unloading and stabilization are achieved by low frequencies. Moreover, learning concerns stable changes in the volume and number in the connectivity of neurons. The higher the volume of synapses during learning in arousal, the higher the number of slow waves is during sleep. Consequently, consolidation is enhanced.

Cognitive function and higher-order processes are influenced by sleep state mechanisms: inertia, homeostasis, and circadian rhythms. According to Burke, et al. [10], circadian rhythms can affect inhibition, while sleep inertia affects visual attention. In addition to that, circadian rhythms and homeostasis regulate reaction time, visual attention maintenance, working memory, mood, alertness, and motivation. In addition, hypo vigilance may cause micro-sleeps that may affect attention dangerously, during various activities. Special monitoring and devices may contribute to the decrease of such incidents [11], [12].

Hypnopaedia is a process of learning during sleep. It has been associated with pop culture and science fiction, in which sleep-learning is presented as easy and automatic. In 1956, Emmons \& Simons (as referred in Oghenero) [13], examined the potential of learning during sleep by EEG and they found that it was not effective. There has been no research interest for years. Since neuroscience has been developed and research on memory has offered a lot of information, the study of Hypnopaedia has started again.

\section{$4 \quad$ Sleep Impact on Learning Process}

Sleep is an enhancive factor for cognitive functions, especially for memory and for cognitive performance in relative tasks. It has been studied through experiments, in which either the duration or the absence of sleep has been controlled. In particular, the effects of sleep deprivation before learning, on memory tasks have been studied. Furthermore, there has been research on the effects of sleep after the learning process, the effects of interventions on sleep patterns, and the repetition of neuron expression during sleep, after learning. Most of these studies claim that sleep is deeply connected to memory processes, in both humans and animals [7].

Neuro-imaging methods during sleep show complex interactions in thalamus circuits, which manage to maintain electro-cortical oscillations in NREM state. The fMRI examination has shown that neuron activity is repeated, in the hippocampus, in the motor cortex, in the dorsomedial, prefrontal cortex, and the left cerebellum. Moreover, reduced activity is observed in the parietal lobes, in the left insula, in the temporal lobes, and the prefrontal area. Neuroplasticity occurs by the interaction of brain cells with the neuron networks and the changes which take place in thalamic and cortical systems. Such results have been primarily derived from experiments conducted on cats [14], [15], [3]. 
From the above data, we conclude that sleep contributes to memory function, thus to learning. In 1801, dream was supposed to affect and change the volume of neuron connections of memory, but the first systematic research was conducted in 1924. This study showed that memory performance was better after a night's sleep. A relevant theory was established and it has been developed in recent years by the support of neurosciences, which offer scientific proof [3].

Research about learning during sleep has been made in infants. Fifer, et al. [16] tested 34 newborns (10 to 73 hours old) with a device which was recording bio-electric brain activity. The researchers applied a sound and a puff on the newborns' eye. In contrast to the control group, infants who were in the experimental group quickly predicted the sequence of stimuli, and the EEG recordings showed maximum, positive slow brainwaves, which are related to memory update. Blinking as a reaction to a stimulus is an indicator of the cerebellar circuit function, which is distinguished to children with autism, dyslexia, ADHD, and schizophrenia. In this way, diagnosis of these disorders may be possible at a very early stage.

Seehagen, et al. [17] examined the effect of sleep on explicit memory, in groups of infants (6 to 12 months old), who had slept for a while or had been deprived of sleep. Infants were examined for their performance in a learning task with puppets, after 4 and 24 hours, respectively. The results showed that infants in the nap condition remembered the activities better than the ones who did not sleep and that after 24 hours the first group had better scores. This study is the first experimental proof of explicit memory consolidation and stabilization in the first year of life.

It is important to note that sleep and sleep functions are not the same through ageing. In newborns, sleep starts by the REM phase, which is $50 \%$ of total sleep and it follows a small cycle of 50 minutes. Until 2 years old, REM sleep is reduced and consists of 20 to $25 \%$ of sleep. NREM sleep is also reduced in adolescence, consisting of $40 \%$ of total sleep and in older ages it decreases too, but in a slower rhythm, especially to the males [2].

Lam, et al. [18] studied how a short nap during the day affects the cognitive performance of preschoolers ( $\mathrm{N}=59,3-5$ years old). They used actigraphs for a week, as well as questionnaires for parents to measure sleep quality, along with tests on attention, vocabulary, and response control. Data analysis showed that a nap is negatively associated with vocabulary, and auditory attention span. If night sleep had been deprived, sleep would have been negatively correlated with cognitive performance and kids who had poor sleep, made more impulsive mistakes. They also noticed that those who had taken a nap during the day slept less at night. This study showed that in this age, a nap deteriorates neurobiological cognitive function -while it is enhancing for adults- and that a night's sleep is crucial for preschoolers' cognitive performance.

So, a nap during the day may be helpful or may have a negative effect, depending on different age groups. Lovato \& Lack [19] argue that a nap decreases sleepiness and improves cognitive performance, only if it lasts for 5 to 15 minutes, and this enhancement has 1 to 3 hours duration. It was sooner proved that even 6 minutes of sleep after learning, enhances memory [20]. This can be attributed to the O Process, in which during a 10-minute-sleep nucleus activity is eliminated and during arousal nucleus are reactivated and vigilance increases. If a nap is 30 minutes or more long, then it is harmful 
for cognitive function right after awakening, but it is also enhancing for many hours, right afterwards. Many hours in vigilance state are related to longer sleep states. According to circadian rhythms, the best time for a nap is early in the afternoon.

Bernier, et al. [21] studied the impact of sleep on executive functions in preschoolers. Mothers of 65 kids filled in a diary of their children's sleep behaviors, since their first year, and a Wechsler Test (WPPSI) was performed 4 years later. The analysis of the results showed that kids who slept enough had better performance in their executive functions, but not better scores in cognitive ability. Researchers underlined the significance of sleep in preschoolers' higher cognitive functions.

Paavonen, et al. [22] studied cognitive performance in 290 children (7, 5 to 8, 8 years old) and related the results to the duration and quality of their sleep. Actigraphs were used for children, as well as special questionnaires about sleeping, which were filled by parents. The researchers examined cognitive ability by four WISC-III subtests along with another two visuo-motor and narrative-memory ability tests. The study results support that less sleep goes along with reduced visuospatial performance, but not less language ability, at this developmental stage.

In another study, researchers [23] measured neuro-behavioral characteristics of 77 students $(\mathrm{M}=10,6$ years old), before and after sleep deprivation or extension, for just an hour. They had previously recorded the children's sleeping patterns for five days. The researchers noticed that even by one-hour deprivation of sleep, the quality of sleep was enhanced. In contrast, an hour of sleep extension caused deterioration in sleep quality. Sleep extension was correlated with shorter reaction time, with better memory ability, whereas in sleep deprivation the abilities were negatively affected. In general, noticeable neuro-behavioral changes occurred and in the sleep deprivation condition executive functions appeared more burdened.

De Bruin, et al. [24] also studied the effects of sleep deprivation or extension to cognitive performance, in a systematic review, which resulted in 16 studies, in the adolescents' population (10 to 19 years old). In a partial sleep deprivation condition, no negative effects were detected in the cognitive ability of adolescents. In both the sleep extension condition and the better-quality sleep condition, memory became stronger. Research results showed that sleep after learning improves memory consolidation. There were a few participants who manifested no clear results. In these studies, many different measures were made, by several tests, and many individual differences were observed. In conclusion, researchers claim that if impaired sleep in teens is enhanced, working memory will also be enhanced, whereas, if teens are deprived of sleep, their ability in vigilant tasks will be reduced.

Araújo \& Almondes [25] conducted a meta-analysis on results about sleep effects on cognitive functions, in children and adolescents. Researchers argue that the biological need for sleep may not be fulfilled, because of everyday routines and special habits. Young children feel sleepy, while teenagers are used to sleeping late at night and watch programs on the internet. This fact results in a decrease of Slow Wave Sleep and delays occur in sleep phases. These delays can hold down visuospatial perception, working memory, and attention. The researchers conclude that poor sleep results in worse cognitive performance, while some children may have better performance by less sleep. It 
is a fact that explicit and procedural memory deteriorates in sleep deprivation conditions.

Kyle, et al. [26] utilized results from a biological bank $(\mathrm{N}=477.529,40$ to 69 years old) extracted by the general population, on the sleep function. The researchers found that 133.314 individuals ( $M=57$ years old) suffered from insomnia. They compared their performance on cognitive ability (reasoning, reaction time, numeric memory, visual memory, etc.) to the performance of others (344.215, M=56 years old) who had no sleep problems. The results showed that those who often suffered from insomnia did slightly better on cognitive tests. Those who took medication for sleep and those who used to sleep less ( $<7$ hours) or more ( $>9$ hours) scored lower. Last, those who slept at night scored higher than those who slept during the day.

It seems that sleep is closely connected to cognitive performance. Researchers are focused on the effects of sleep on memory, executive function tasks, and learning. A good sleep before or after the learning process enhances learning outcomes. In contrast, sleep deprivation, even a minus one, may cause negative effects in executive functions, in attention maintenance, and long-term memory, according to a meta-analysis [27]. The above effects depend on age, sex, time of sleep, the subjective sense of sleepiness, and the delay of sleep.

In addition, several practices during sleep may empower learning. These techniques consist of olfactory or audio stimuli that reactivate memories derived from the learning process. This is usually accomplished by electrical or audio stimulation or by drugs such as noradrenalin and glutamine [28], [29]. In fact, sensory stimuli may become conditional ones. Many studies have deployed the sense of hearing or smelling during sleep for learning, since these senses are still active during sleep [30], [31], [32]. An audio stimulus can activate twice the auditory cortex and the thalamus in the NREM phase. Calling someone's name can also activate the right amygdala and the prefrontal cortex. The sound of clicks during sleep can boost problem-solving ability [7].

Moreover, learning procedures affect sleep. Research on animals as well as humans prevails that there is an increase in Slow Wave Sleep, in oscillations in the hippocampus, which produce consolidated information and knowledge. REM sleep phases are increased too, and this enhances memory consolidation in the long -term memory [33], [34].

In short, sleep is a function that differs among ages, and its effect on cognition and performance varies. Newborns can learn even during sleep. Children are positively affected by a good night's sleep. Adults may benefit from a short nap during the day. Adolescents have poor quality sleep, which affects their cognitive performance and especially their executive functions in a negative way. People who are over 40 years old and suffer from insomnia are not affected negatively, as far as their cognitive abilities are concerned, but insufficient sleep deteriorates cognition, in general.

\section{Conclusion}

Sleep seems to be a mysterious and dynamic function. Since the development of neurosciences, multiple procedures come to light which take place in a motionless 
body. Why do people sleep? Is it because circadian rhythms force us to do so? Is it because the organism needs some time to be neuro-biologically balanced in order to survive? Is it because a lot of information that we come across every day must be processed and organized? Is it because some functions are manifested only during dreams?

According to neuroscience, sleep is presented by oscillations, peaks, electric power, periods, time, motion, and neurotransmitters, all of which differ in individuals and each developmental stage. Babies sleep more and dream more. It is possible for them to learn during sleep and to recall better after a good sleep.

As children grow up, in preschool years, daytime becomes a field for activity and education. If a short nap mediates during this time, it can affect the vocabulary performance and the function of attention in a negative manner. In contrast, a night sleep favors the consolidation of data extracted from an interesting and explorative previous day.

Children and adolescents sleep, but neither enough nor sufficiently. Their sleep is of poor quality because of everyday habits, such as overusing screens (pc, TV, mobile phones, etc.) and routines such as going to school very early. This affects their cognitive performance. Perhaps we should consider appropriate changes that could lead to sleep restoration and better cognitive performance. In addition, adolescents dream more and sleep less. Developmentally, the youth seems to need more vision and more action.

Adults benefit from a nap during the day even if it lasts for 6 minutes. A nap early in the afternoon can recharge nucleus in the brain effectively and achieve the necessary level of vigilance.

People who are above 55 years old use to sleep less and gradually Slow Wave Sleep becomes less, too. They usually deal with insomnia, which nevertheless, does not affect much of their cognitive function. In general, cognitive performance may be decreased by insomnia or sleeping during the daytime, because of sleep deprivation.

Sleep appears to evolve while getting older. Is it because it may relate to maturation and neuro-plasticity? It seems that the more immature or unshaped one is, the more active one's sleep is. Also, the more mature and 'wise' one is, the shorter one's sleep is.

We should consider how to utilize these results about cognitive enhancement or even about interventions in special education. Researchers have already made use of technologies which are put into action during sleep and they boost memory and learning. New research is necessary to be conducted on what, how, and for whom these interventions may upgrade learning, as well as the quality of life.

\section{$6 \quad$ References}

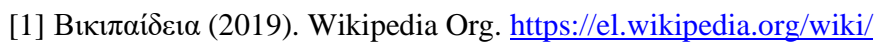

[2] Carskadon, M. A., \& Dement, W. C. (2011). Monitoring and staging human sleep. In M. H. Kryger, T. Roth, \& W. C. Dement (Eds.), Principles and practice of sleep medicine, 5th edition, (p. pp 16-26). Elsevier Saunders. https://doi.org/10.1016/b978-1-4160-6645-3.000 $\underline{02-5}$

[3] Walker, M. P. (2008). Cognitive consequences of sleep and sleep loss. Sleep Medicine, 9(1), S29-S34. https://doi.org/10.1016/s1389-9457(08)70014-5 
[4] Perogamvros, L., Dang-Vu, T. T., Desseilles, M., \& Schwartz, S. (2013). Sleep and dreaming are for important matters. Frontiers in Psychology, 4(474). https://doi.org/10.3389/fps yg.2013.00474

[5] Tourimpampa A., Drigas A. \& Economou A. (2017). Relation of Memory and Linguistic Fields and ICT Tools for Memory and Language Comprehension. iJES,5 (1), pp:4-15. https://doi.org/10.3991/ijes.v5i1.6275

[6] Walker, M. P, \& Stickgold, R. (2006). Sleep, Memory, and Plasticity. Annual Review of Psychology, 57(1), 139-166. https://doi.org/10.1146/annurev.psych.56.091103.070307

[7] Peigneux, P., Laureys, S., Delbeuck, X., \& Maquet, P. (2001). Sleeping brain, learning brain. The role of sleep for memory systems. Neuroreport, 12(18), A111-A124. https:// doi.org/10.1097/00001756-200112210-00001

[8] Budzynski, T. H. (2011). Twilight Learning Revisited1. Biofeedback, 39(4), 155-166. https://doi.org/10.5298/1081-5937-39.4.08

[9] Tononi, G, \& Cirelli, C. (2003). Sleep and synaptic homeostasis: a hypothesis. Brain Research Bulletin, 62(2), 143-150. https://doi.org/10.1016/j.brainresbull.2003.09.004

[10] Burke, T. M., Scheer, F. A. J. L., Ronda, J. M., Czeisler, C. A, \& Wright, K. P. (2015). Sleep inertia, sleep homeostatic and circadian influences on higher-order cognitive functions. Journal of Sleep Research, 24(4), 364-371. https://doi.org/10.1111/jsr.12291

[11] Akrout B. \& Mahdi W. (2013). Hypovigilance Detection Based on Eyelids Behavior Study. iJES, 1(1), pp:39-45. https://doi.org/10.3991/ijes.v1i1.2927

[12] Jemai O., Teyeb I., Bouchrika T.and Ben Amar C. (2013). A Novel Approach for Drowsy Driver Detection Using Eyes Recognition System Based on Wavelet Network. iJES, 1(1), pp: 46-52. https://doi.org/10.3991/ijes.v1i1.2929

[13] Oghenero, O.A. (2019). A Visuo-Spatial Understanding of Hypnopaedia. International Journal of Innovative Science and Research Technology, 4(1). https://ijisrt.com/wp-content/uploads/2019/01/IJISRT19JA205.pdf

[14] Frank, M. G., Issa, N. P, \& Stryker, M. P. (2001). Sleep Enhances Plasticity in the Developing Visual Cortex. Neuron, 30(1), 275-287. https://doi.org/10.1016/s0896-6273(01)00 $\underline{279-3}$

[15] Hobson, J. A., \& Pace-Schott, E. F. (2002). The cognitive neuroscience of sleep: neuronal systems, consciousness and learning. Nature Reviews Neuroscience, 3(9), 679-693. https://doi.org/10.1038/nrn915

[16] Fifer, W. P., Byrd, D. L., Kaku, M., Eigsti, I.-M., Isler, J. R., Grose-Fifer, J., Tarullo, A. R., \& Balsam, P. D. (2010). Newborn infants learn during sleep. Proceedings of the National Academy of Sciences of the United States of America, 107(22), 10320-10323. https://doi.org/10.1073/pnas.1005061107

[17] Seehagen, S., Konrad, C., Herbert, J. S., \& Schneider, S. (2015). Timely sleep facilitates declarative memory consolidation in infants. Proceedings of the National Academy of Sciences of the United States of America, 112(5), 1625-1629. https://doi.org/10.1073/pnas. $\underline{1414000112}$

[18] Lam, J. C., Mahone, E. M., Mason, T, \& Scharf, S. M. (2011). The Effects of Napping on Cognitive Function in Preschoolers. Journal of Developmental \& Behavioral Pediatrics, 32(2), 90-97. https://doi.org/10.1097/dbp.0b013e318207ecc7

[19] Lovato, N, \& Lack, L. (2010). The effects of napping on cognitive functioning. Progress in Brain Research, 185, 155-166. https://doi.org/10.1016/b978-0-444-53702-7.00009-9

[20] Lahl, O., Wispel, C., Willigens, B, \& Pietrowsky, R. (2008). An ultra-short episode of sleep is sufficient to promote declarative memory performance. Journal of Sleep Research, 17(1), 3-10. https://doi.org/10.1111/j.1365-2869.2008.00622.x 
[21] Bernier, A., Beauchamp, M. H., Bouvette-Turcot, A.-A., Carlson, S. M, \& Carrier, J. (2013). Sleep and Cognition in Preschool Years: Specific Links to Executive Functioning. Child Development, 84(5), 1542-1553. https://doi.org/10.1111/cdev.12063

[22] Paavonen, E. J., Räikkönen, K., Pesonen, A.-K., Lahti, J., Komsi, N., Heinonen, K., Järvenpää, A.-L., Strandberg, T., Kajantie, E, \& Porkka-Heiskanen, T. (2010). Sleep quality and cognitive performance in 8-year-old children. Sleep Medicine, 11(4), 386-392. https:// doi.org/10.1016/j.sleep.2009.09.009

[23] Sadeh, A., Gruber, R, \& Raviv, A. (2003). The Effects of Sleep Restriction and Extension on School-Age Children: What a Difference an Hour Makes. Child Development, 74(2), 444-455. https://doi.org/10.1111/1467-8624.7402008

[24] de Bruin, E. J., van Run, C., Staaks, J., \& Meijer, A. M. (2017). Effects of sleep manipulation on cognitive functioning of adolescents: A systematic review. Sleep Medicine Reviews, 32, 45-57. https://doi.org/10.1016/j.smrv.2016.02.006

[25] Araújo, D. de F, \& Almondes, K. M. de. (2013). Sleep and cognitive performance in children and pre-adolescents: a review. Biological Rhythm Research, 45(2), 193-207. https:// doi.org/10.1080/09291016.2013.790136

[26] Kyle, S. D., Sexton, C. E., Feige, B., Luik, A. I., Lane, J., Saxena, R., Anderson, S. G., Bechtold, D. A., Dixon, W., Little, M. A., Ray, D., Riemann, D., Espie, C. A., Rutter, M. K., \& Spiegelhalder, K. (2017). Sleep and cognitive performance: cross-sectional associations in the UK Biobank. Sleep Medicine, 38, 85-91. https://doi.org/10.1016/j.sleep.2017. $\underline{07.001}$

[27] Lowe, C. J., Safati, A, \& Hall, P. A. (2017). The neurocognitive consequences of sleep restriction: A meta-analytic review. Neuroscience \& Biobehavioral Reviews, 80, 586-604. https://doi.org/10.1016/j.neubiorev.2017.07.010

[28] Diekelmann, S. (2014). Sleep for cognitive enhancement. Frontiers in Systems Neuroscience, 8(46). https://doi.org/10.3389/fnsys.2014.00046

[29] Cellini, N., \& Mednick, S. C. (2019). Stimulating the sleeping brain: Current approaches to modulating memory-related sleep physiology. Journal of Neuroscience Methods, 316, 125 -136. https://doi.org/10.1016/j.jneumeth.2018.11.011

[30] Shanahan, L. K, \& Gottfried, J. A. (2014). Olfactory Insights into Sleep-Dependent Learning and Memory. Progress in Brain Research, 309-343. https://doi.org/10.1016/b978-0-44463350-7.00012-7

[31] Batterink, L. J., Creery, J. D, \& Paller, K. A. (2016). Phase of Spontaneous Slow Oscillations during Sleep Influences Memory-Related Processing of Auditory Cues. Journal of Neuroscience, 36(4), 1401-1409. https://doi.org/10.1523/jneurosci.3175-15.2016

[32] Andrillon, T., Pressnitzer, D., Léger, D., \& Kouider, S. (2017). Formation and suppression of acoustic memories during human sleep. Nature Communications, 8(1). https://doi.org/ 10.1038/s41467-017-00071-z

[33] Eschenko, O., Ramadan, W., Molle, M., Born, J., \& Sara, S. J. (2008). Sustained increase in hippocampal sharp-wave ripple activity during slow-wave sleep after learning. Learning \& Memory, 15(4), 222-228. https://doi.org/10.1101/lm.726008

[34] Smith, C, \& Lapp, L. (1991). Increases in number of REMS and REM density in humans following an intensive learning period. Sleep, 14(4), 325-330. https://doi.org/10.1093/ $\underline{\text { sleep/14.4.325 }}$ 


\section{Authors}

Sevasti Kapsi is with Institute of Informatics and Telecommunications - Net Media Lab \& Mind-Brain R\&D, Agia Paraskevi, 153 10, Athens, Greece (e-mail: sevastikapsi@gmail.com).

Spyridoula Katsantoniis with Institute of Informatics and Telecommunications Net Media Lab \& Mind-Brain R\&D, Agia Paraskevi, 153 10, Athens, Greece (e-mail: skatsantoni@gmail.com).

Athanasios Drigas is a Research Director at N.C.S.R. 'Demokritos', Institute of Informatics and Telecommunications - Net Media Lab \& Mind-Brain R\&D, Agia Paraskevi, 153 10, Athens, Greece (e-mail: dr@iit.demokritos.gr).

Article submitted 2020-07-17. Resubmitted 2020-07-27. Final acceptance 2020-07-28. Final version published as submitted by the authors. 